\title{
A HEART FOR RUNNING - SAFETY CONSIDERATIONS ON AEROBIC EXERCISE IN THE SUBACUTE PHASE OF STROKE - DATA FROM THE PHYS-STROKE TRIAL
}

Rackoll T1,2,5*; Nave $A^{1-4}$; Grittner $\mathrm{U}^{1,6}$; Bläsing $\mathrm{H}^{7}$; Gorsler $\mathrm{A}^{8}$; Nabavi $\mathrm{D}^{9}$; Audebert $\mathrm{H}^{1,2}$;

Steinhagen-Thiessen $E^{10}$; Meisel $A^{1,2,5}$; Endres $M^{1-4,11}$; Hesse $S^{\dagger, 12}$; Ebinger $\mathbf{M}^{1,12}$; and Flöel $A^{1,13}$

${ }^{1}$ Charité - Universitätsmedizin Berlin, Center for Stroke Research Berlin; ${ }^{2}$ Charité - Universitätsmedizin Berlin, Klinik und Hochschulambulanz für Neurologie;

${ }^{3}$ German Center for Cardiovascular Research, partner site Berlin; ${ }^{4}$ Berlin Institute of Health $(\mathrm{BIH}) ;{ }^{5}$ Charité - Universitätsmedizin Berlin, NeuroCure

Clinical Research Center; ${ }^{6}$ Charité - Universitätsmedizin Berlin, Institute of Biometry and Clinical Epidemiology; ${ }^{7}$ Median Klinik Gruenheide, Gruenheide;

${ }^{8}$ Kliniken Beelitz GmbH, Beelitz-Heilstaetten; ${ }^{9} \mathrm{Clinic}$ for Neurology, Vivantes Klinikum Neukoelln, Berlin; ${ }^{10}$ Evangelisches Geriatriezentrum Berlin gGmbH;

${ }^{11}$ German Center for Neurodegenerative Diseases, partner site Berlin; ${ }^{12}$ Medical Park Berlin Humboldtmuehle; ${ }^{13}$ Department of Neurology, University Medicine Greifswald * Corresponding author: torsten.rackoll@charite.de

\section{Introduction}

Within the subacute phase of stroke aerobic exercise including treadmill training is a promising rehabilitative therapy (Saunders et al, 2016) to improve walking abilities and minimize physical deterioration but its safety still needs to be further analyzed. Our aim was to identify patients from the trial "Physical fitness in subacute stroke - PHYSStroke" with an increased risk of encountering a serious adverse event (SAE) during or after an aerobic exercise intervention in the subacute phase of stroke.

\section{Methods}

Data are drawn from the German endpointblinded, multicenter, randomized clinical trial 'Physical Activity in Subacute Stroke (PHYS-Stroke)' (Flöel et al, 2014). In this trial patients in the early subacute phase of stroke (day 5-45 after stroke) were recruited from seven rehabilitation clinics in and around Berlin. Patients were randomized to receive 25 minutes of either treadmillbased, aerobic physical exercise (PHYS) or relaxation sessions (RELAX) five times per week for four weeks, in addition to standard rehabilitative therapy.

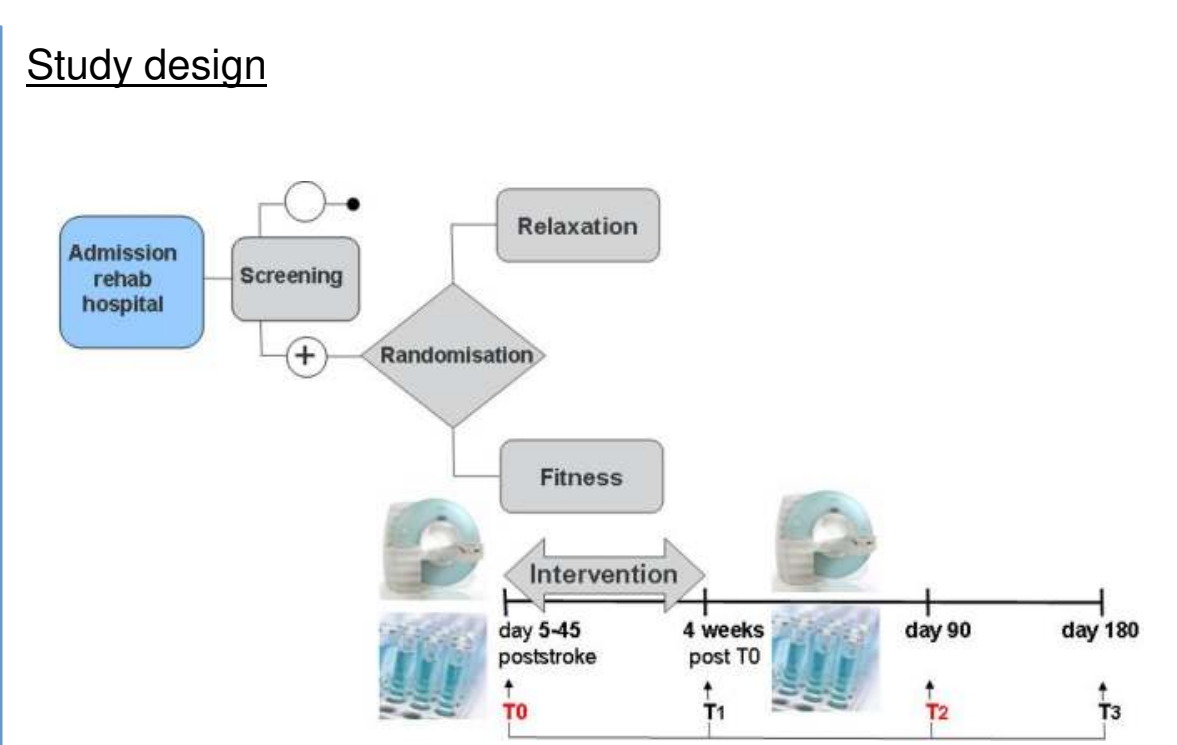

Results

Safety analyzes are on the basis of 200 patients who were randomized in the trial (end of recruitment: April 30 th 2017 ; Database closed: Nov 10 th 2017 )

\begin{tabular}{|l|l|l|l|l|l|}
\hline & $\begin{array}{l}\text { All } \\
\mathbf{n = 2 0 0}\end{array}$ & $\begin{array}{l}\text { PHYS } \\
\mathbf{n = 1 0 5}\end{array}$ & $\begin{array}{l}\text { RELAX } \\
\mathbf{n = 9 5}\end{array}$ & $\begin{array}{l}\text { Odds Ratio } \\
(\mathbf{9 5 \%} \mathrm{Cl})\end{array}$ & $\boldsymbol{p}$ \\
\hline Stroke, $\mathrm{n}[\%]$ & $14[7 \%]$ & $10[10 \%]$ & $4[4 \%]$ & $\begin{array}{l}2.40 \\
(0.73-7,91)\end{array}$ & 0.15 \\
\hline $\begin{array}{l}\text { Myocardial } \\
\text { infarction }\end{array}$ & 0 & 0 & 0 & & \\
\hline $\begin{array}{l}\text { Admission to } \\
\text { hospital, } \mathrm{n}[\%]\end{array}$ & $26[13 \%]$ & $19[18 \%]$ & $7[7 \%]$ & $\begin{array}{l}2.78 \\
(1.11-6.94)\end{array}$ & 0.03 \\
\hline Death, $\mathrm{n}[\%]$ & $5[3 \%]$ & $1[1 \%]$ & $4[4 \%]$ & $\begin{array}{l}0.22 \\
(0.02-1.99)\end{array}$ & 0.18 \\
\hline
\end{tabular}

- $57 \%(n=8)$ of recurrent strokes and $35 \%(n=9)$ of readmission to hospital happened during intervention phase

- SAEs happening in the PHYS group lead to a worse health status in $34 \%$ compared to $23 \%$ in the RELAX group (PHYS $n=32, \operatorname{RELAX~} n=$ 13 , not significant)

- Patients with recurrent strokes were more often older, females with an early intervention start compared to the entire trial population

- Recurrent strokes within the intervention phase happend in the PHYS group $(p=0.01)$ and were older than 75 years of age and predominantly female $(75 \%)$

- Patients with readmission to hospital during intervention phase were more likely to have atrial fibrillation $(56 \%, p=0.03)$ and/or a stroke form with a cardioembolic aetiology $(56 \%, p=0.03)$

- No myocardial infarction were reported

- Death happened more often in the RELAX group but none were regarded as related to the intervention by the data safety monitoring board

- Self-reported falls occured more often in the PHYS group $(n=36)$ than in the RELAX group $(n=14, p=0.01)$ but had no serious consequences

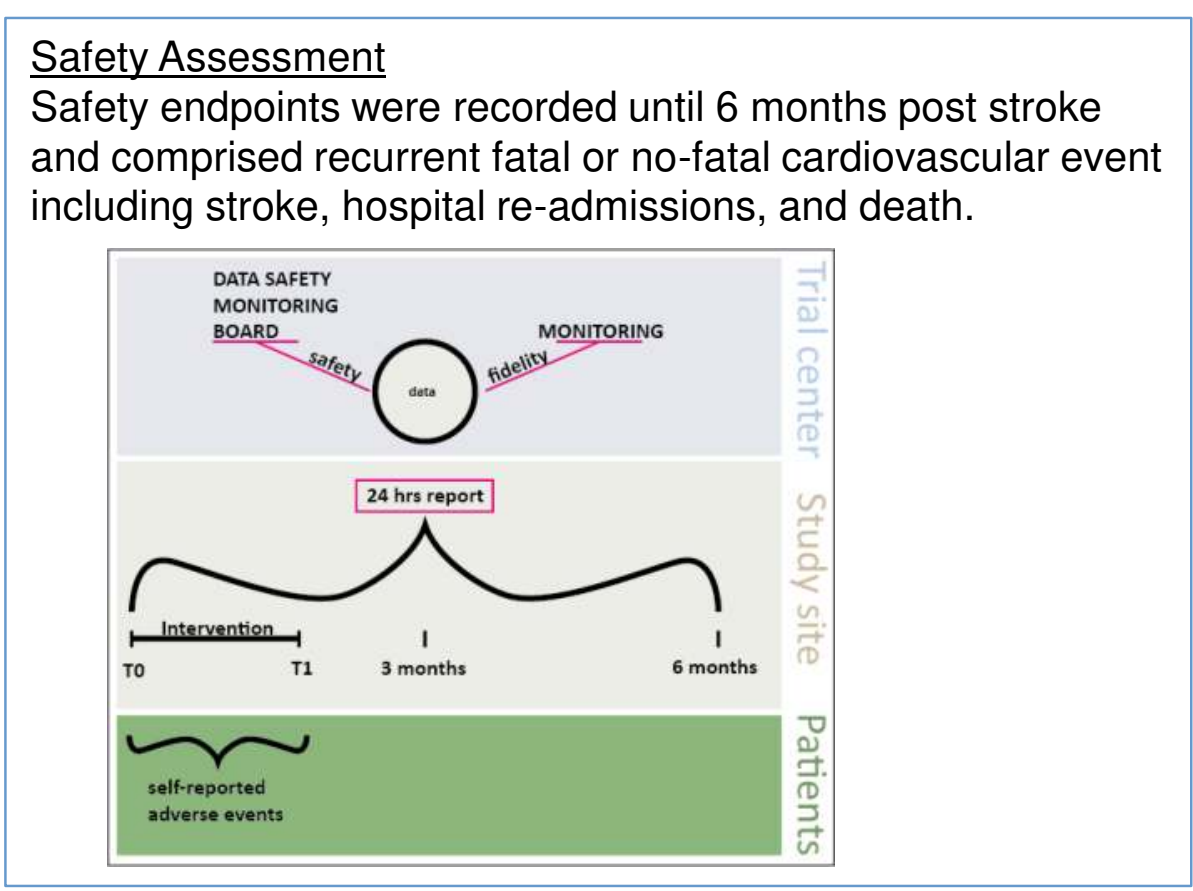

Discussion

Despite its many advantages to prevent from secondary events an aerobic fitness intervention might provoke further complications in older females or in patients with cardiac comorbidities in the subacute phase of stroke and should be administered with caution or in the later phase of recovery. An increased occurence of falls has been reported by other trials and warrants further assessment to prevent from fractures or trauma.

Saunders DH, Sanderson M, Brazzelli M, et al. Physical fitness training for stroke patients. Cochrane Database Syst Rev. 2016;10(3)

Flöel A, Werner C, Grittner U, et al. Physical fitness training in Subacute Stroke (PHYS-STROKE)--study protocol for a randomised controlled trial. Trials. 2014;15:45 